\title{
Constraining the Milky Way mass with Hypervelocity Stars
}

\author{
G. Fragione ${ }^{\mathrm{a}, *}$ A. Loeb $^{\mathrm{b}}$ \\ ${ }^{a}$ Department of Physics, Sapienza, University of Rome, P.le A. Moro 2, Roma, Italy \\ ${ }^{b}$ Astronomy Department, Harvard University, 60 Garden St., Cambridge, MA 02138, USA
}

\begin{abstract}
Although a variety of techniques have been employed for determining the Milky Way dark matter halo mass distribution, the range of allowed masses spans both light and heavy values. Knowing the precise mass of our Galaxy is important for placing the Milky Way in a cosmological $\Lambda$ CDM context. We show that hypervelocity stars (HVSs) ejected from the center of the Milky Way galaxy can be used to constrain the mass of its dark matter halo. We use the asymmetry in the radial velocity distribution of halo stars due to escaping HVSs, which depends on the halo potential (escape speed) as long as the round trip orbital time is shorter than the stellar lifetime, to discriminate between different models for the Milky Way gravitational potential. Adopting a characteristic HVS travel time of 330 Myr, which corresponds to the average mass of main sequence HVSs, we find that current data favors a mass for the Milky Way in the range $(1.2-1.9) \times 10^{12} \mathrm{M}_{\odot}$.
\end{abstract}

Keywords: Galaxy: halo, Galaxy: kinematics and dynamics, stars: kinematics and dynamics

\section{Introduction}

Hypervelocity Stars (HVSs) are defined as stars able to escape the gravitational well of the Milky Way (MW). Theoretically predicted by Hills (1988) as the consequence of interactions of binary stars with the massive Black Hole $(\mathrm{BH})$ in the Galactic Centre (GC), HVSs were first observed by Brown et al. (2005). More then 20 HVSs at distances between 50 and $120 \mathrm{kpc}$ from the GC, and velocities up to $\approx 700 \mathrm{~km} \mathrm{~s}^{-1}$, have been found (Multiple Mirror Telescope (MMT) spectroscopic survey (Brown et al., 2010, 2014)). A similar number of bound HVSs, i.e. stars ejected by the same mechanism of unbound stars, but with velocities below the Galactic escape speed, have been observed (Brown et al., 2007a,b, 2014). The MMT targets stars with the magnitudes and colors of $2.5-4 \mathrm{M}_{\odot}$ late B-type stars, since they should not exist at faint magnitudes in the outer halo far from star-forming regions unless they were ejected to that location. Given the MMT target selection (Brown et al., 2014), the sample stars could be either Main Sequence (MS) B stars, evolved Blue Horizontal Branch (BHB) stars or blue stragglers, while only a few of them have a defined stellar type. Recent studies have started to investigate low-mass HVS candidates (Palladino et al., 2014; Li et al., 2015; Ziegerer et al., 2015). The physical mechanisms responsible for the production of the observed HVSs are still debated. However, due to their extreme velocities, the origin of HVSs involves strong dynamical interactions probably with a single or binary $\mathrm{BHs}$

\footnotetext{
*Corresponding author

Email addresses: giacomo.fragione@uniroma1.it

(G. Fragione), aloeb@cfa.harvard.edu (A. Loeb)
}

in the GC (Yu \& Tremaine, 2003; Ginsburg \& Loeb, 2006, 2007; O'Leary \& Loeb, 2008; Sari et al., 2009; Ginsburg et al., 2012; Capuzzo-Dolcetta \& Fragione, 2015; Fragione \& Capuzzo-Dolcetta, 2016; Fragione \& Ginsburg, 2016; Fragione et al., 2016) or in a nearby galaxy (Gualandris \& Portegies Zwart, 2007; Sherwin et al., 2008; Boubert \& Evans, 2016).

The study of HVSs can provide clues about the process responsible for their production in the Galactic Centre region (Gould \& Quillen, 2003). As their orbits are completely determined by the MW potential, Gnedin et al. (2005) and Yu \& Madau (2007) suggested to use the kinematics of HVSs to probe the Galactic potential triaxiality. Moreover, HVSs can be used to constrain the mass distribution of our Galaxy, which is still highly uncertain. Gnedin et al. (2010) used the MMT sample to constrain the Galactic mass profile out to $80 \mathrm{kpc}$. In this paper we apply the method proposed by Perets et al. (2009) to discriminate among different Galactic potential models, with a focus on measuring the total dark halo mass.

A variety of techniques have been employed for determining the MW dark matter halo mass distribution (Gnedin et al., 2010; McMillan, 2011; Eadie et al., 2015; Wang et al., 2015), but, nevertheless, the range of allowed values spans both light $\left(\lesssim 10^{12} \mathrm{M}_{\odot}\right)$ and heavy $\left(\gtrsim 2 \times 10^{12} \mathrm{M}_{\odot}\right)$ values (Wang et al., 2015). Several classes of kinematic tracers have been used for this purpose, but suffer from systematics caused by the lack of reliable tangential velocity and distance measurements (Gibbons et al., 2014). Küpper et al. (2015) and Bovy et al. (2016) used the tidal stream of the Milky Way's star clusters Palomar 5 and GD-1 to probe the 
shape of the Galactic potential. Tidal streams are useful tracers of the Galactic mass in the inner halo. On the other hand, HVSs could be highly valuable in discriminating among different mass models out to very large Galactocentric distances, in particular once precise data on their proper motions will be available from Gaia (Perets et al., 2009).

Knowing the precise mass of our Galaxy is important for placing the $\mathrm{MW}$ in a cosmological $\Lambda \mathrm{CDM}$ context. Although the difference in mass between light and heavy halo masses is just by a factor of $2-3$, such a factor leads to a major difference in the conversion efficiency of baryons into stars (higher for lighter haloes), places the Large Magellanic Cloud (Kallivayalil et al., 2013) and the Leo I dwarf spheroidal (Boylan-Kolchin et al., 2013) on unbound (light halo) or bound (heavy halo) orbits and can or cannot solve the Too-Big-to-Fail problem (Taylor et al., 2015). This latter problem is one of the two prominent challenges concerning the satellite galaxies in the MW and consists in the fact that the most massive subhaloes of numerical simulations, which in typical galaxy formation models would host the most luminous satellites, are too dense to be dynamically consistent with observations of any of the known MW companions (Boylan-Kolchin et al., 2011). The other challenge is the so-called Missing Satellite Problem, as $\Lambda$ CDM model predicts hundred of subhaloes but a smaller set of galaxies is observed (Klypin et al., 1999). Several possible solutions have been suggested, including uncertainties in the mass of the MW halo (Guo et al., 2015; Kang X. et al., 2016).

In this paper, we study the kinematics of HVSs in the Galaxy as a probe of the MW halo mass. The outline of the paper is as follows. In Section 2, we describe the method we use to discriminate among different halo masses. In Section 3 we present our models for the MW gravitational potential. In Section 4, we perform numerical simulations of HVSs motion to study their kinematics and provide results. Finally, in Section 5, we summarize our main conclusions.

\section{Method}

Theoretical calculations by Yu \& Tremaine (2003) suggest that the HVSs ejection rate, both in case the source at the GC is a single or a binary $\mathrm{BH}$, is $\approx 10^{-5}-10^{-4}$ $\mathrm{yr}^{-1}$. If HVSs ejections are continuous and isotropic, their number density is (Brown, 2015)

$$
n(r)=\frac{d N / d t}{4 \pi r^{2} d r / d t} \approx \frac{8}{(r / \mathrm{kpc})^{2}} \mathrm{kpc}^{-3},
$$

implying that HVSs are rare objects. The MMT survey (Brown et al., 2014) targeted stars luminous enough to be observed in the Galactic halo where the relative number of HVSs is expected to be higher. Deason et al. (2014) found evidence for a very steep outer halo density profile, implying that the relative frequency of HVSs is much higher in the outer halo than in the inner halo. Moreover, at Galactic latitudes $|b| \gtrsim 30^{\circ}$, the survey is less likely to be contaminated by the disk and runaway stars (Bromley et al., 2009; Kenyon et al., 2014).

We assume that HVSs are ejected from the GC (Bromley et al., 2006), and escape the MW if the ejection velocity $v_{e j}$ is higher than the local escape speed $v_{e s c}(r)$, which depends on the Galactic potential. While unbound stars will leave the MW, bound stars will reach the apocentre of their orbit and then return back to the $\mathrm{GC}$ with a negative radial velocity (Bromley et al., 2009; Kenyon et al., 2008; Brown, 2015). The observations reveal a significant asymmetry in the tail of the velocity distribution of the sample stars. In particular, there is a significant lack of stars with $v_{r}<-275 \mathrm{~km} \mathrm{~s}^{-1}$ in Galactocentric coordinates (Brown et al., 2007a,b, 2010). We divide the stars of the sample to outgoing stars with positive radial velocities in Galactocentric coordinates, and ingoing stars with negative radial velocities. Perets et al. (2009) proposed a method that uses the observed asymmetry between ingoing and outgoing stars to discriminate among different Galactic potential models. Such asymmetry originates both from the MW gravitational potential as well as from the finite lifetime of HVSs.

Bound stars can be spotted either as outgoing stars or ingoing stars, according to when they are observed in their orbit (Kenyon et al., 2014). However, unbound HVSs can be observed only as outgoing. Therefore, an asymmetry in the distribution of ingoing and outgoing stars is expected if HVSs are continuously ejected from the GC (Perets et al., 2009). Whereas bound stars are expected to be symmetrically divided between ingoing and outgoing stars, unbound HVSs can have only positive velocities. Furthermore, while unbound HVSs are not limited in ejection velocity, except for the limitations of the assumed ejection model, the bound stars must satisfy $v_{e j}<v_{e s c}(r)$. At a given Galactocentric distance $r$, ingoing stars can be observed with a negative velocity whose amplitude is at maximum $v_{e s c}(r)$, which depends on the Galactic gravitational potential. As consequence, for a given MW model, no ingoing stars are expected to be found below the curve $-\nu(r)=-v_{\text {esc }}(r)$ in the v-r plane. Therefore, the asymmetric distance-velocity distribution can be used to directly constrain the Galactic potential (Perets et al., 2009).

However, some stars may disappear from view because they evolve to a different stellar type (Kenyon et al., 2008; Bromley et al., 2009). For example, the finite lifetime of MS stars goes like $t_{*} \propto m^{-\alpha}$, with $\alpha \approx 3$, implies that massive stars ejected from the GC cannot reach large Galactocentric distances and fall back toward the GC before leaving the MS (Bromley et al., 2006). The MMT targeted stars that could be late-type MS B stars with masses in the range $2.5-4 \mathrm{M}_{\odot}$, for which the maximum travel time would be $t_{*} \approx 1-6 \cdot 10^{8} \mathrm{yr}$ (Brown et al., $2010,2014)$. The asymmetry in the velocity-distance distribution is still expected but the cutoff $-\nu(r)$ will also depend on the finite travel time. Moreover, stars of different 
types have different travel times and will lead to distinct distance-velocity cutoffs, providing independent probes of the Galactic potential (Perets et al., 2009).

In conclusion, different Galactic potential models give different $v_{e s c}(r)$, which, combined together with different travel times, lead to peculiar cutoffs in the v-r plane. In this paper we apply the method proposed by Perets et al. (2009) to current data on halo stars, with a focus on measuring the dark halo mass. We draw critical lines for HVSs both as function of the dark halo mass $M_{D M}$ and of the stars travel time $t_{*}$. Whereas Perets et al. (2009) look for the best fit model that shows the largest asymmetry, we consider the one that gives compatible asymmetric distribution of stars $\Delta$ and number of high-velocity outliers $\Gamma$ in the MMT sample (see Section 4 for details) to constrain the MW mass.

\section{Models for the MW gravitational potential}

As described in the previous section, HVSs data can be used to constrain the MW potential. We describe the MW potential with a 4-component model $\Phi(r)=\Phi_{B H}+$ $\Phi_{b u l}(r)+\Phi_{d i s k}(r)+\Phi_{N F W}(r)$ (Kenyon et al., 2008, 2014), including the potential of the central $\mathrm{BH}$

$$
\Phi_{B H}(r)=-\frac{G M_{B H}}{r}
$$

where $M_{B H}=4 \times 10^{6} \mathrm{M}_{\odot}$, the bulge

$$
\Phi_{b u l}(r)=-\frac{G M_{b u l}}{r+a}
$$

where $M_{b u l}=3.76 \times 10^{9} \mathrm{M}_{\odot}$ and $a=0.1 \mathrm{kpc}$, the disk

$$
\Phi_{d i s k}(R, z)=-\frac{G M_{d i s k}}{\left.\sqrt{\left(R^{2}+\left(b+\sqrt{c^{2}+z^{2}}\right)^{2}\right.}\right)},
$$

where $M_{d i s k}=5.36 \times 10^{10} \mathrm{M}_{\odot}, b=2.75 \mathrm{kpc}$ and $c=0.3$ $\mathrm{kpc}$, and the dark matter halo

$$
\Phi_{N F W}(r)=-\frac{G M_{D M} \ln \left(1+r / r_{s}\right)}{r} .
$$

While the parameters of the baryonic components $(\mathrm{BH}$ + Bulge + Disk) of the potential are kept fixed, the mass parameter (not to be confused with the total halo mass) of the Navarro-Frenk-White (NFW) dark halo $M_{D M}$ (Navarro et al., 1997) is varied in the range $(0.6-1.8) \times 10^{12} \mathrm{M}_{\odot}$. The scale radius $r_{s}$ changes accordingly so that the Galactic circular velocity at Sun's distance $(8.15 \mathrm{kpc})$ would be 235 $\mathrm{km} \mathrm{s}^{-1}$ (Reid et al., 2014), while the virial radius $r_{200}$ is defined as the radius within which the enclosed average density is 200 times the mean matter density in the Universe. The concentration is defined as $C=r_{200} / r_{s}$; see Table 1 for the cases under consideration.

\begin{tabular}{|cccc|}
\hline$M_{D M}\left(10^{12} \mathrm{M}_{\odot}\right)$ & $r_{s}(\mathrm{kpc})$ & $r_{200}(\mathrm{kpc})$ & $C$ \\
\hline 0.6 & 14.4 & 221.2 & 17.4 \\
0.8 & 17.4 & 233.8 & 13.5 \\
1.0 & 20.0 & 248.6 & 12.4 \\
1.2 & 22.4 & 261.3 & 11.7 \\
1.4 & 24.6 & 272.6 & 11.1 \\
1.6 & 26.6 & 282.8 & 10.6 \\
1.8 & 28.5 & 292.0 & 10.2 \\
\hline
\end{tabular}

Table 1: Parameters of the MW's NFW Dark Halo
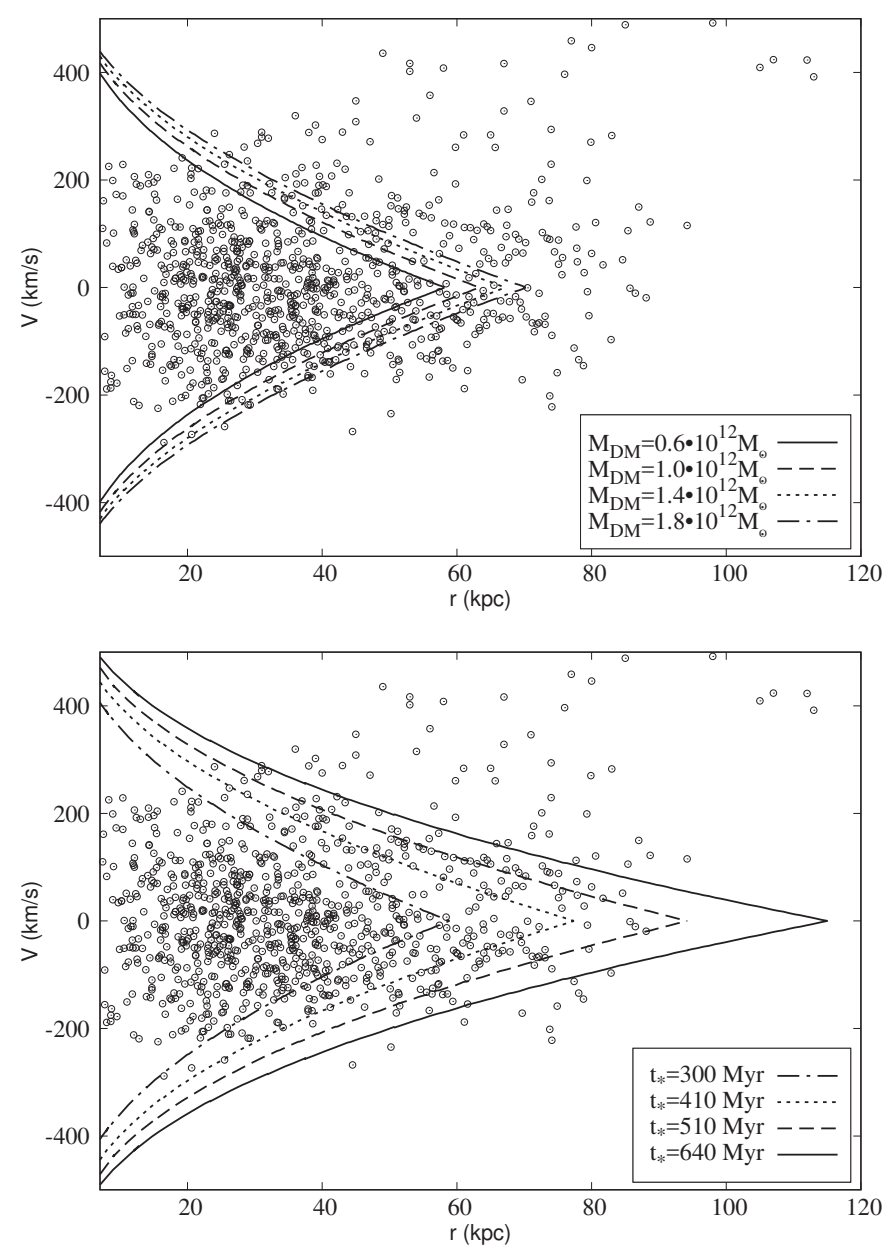

Figure 1: Critical lines for different halo masses (top) and star travel times (bottom). In the top panel, the HVSs travel time is fixed to $t_{*}=330 \mathrm{Myr}$, while, in the bottom panel, the mass parameter of the NFW dark halo $M_{D M}$ is set to $10^{12} \mathrm{M}_{\odot}$. Circles are observed distances and velocities of the MMT star sample (Brown et al., 2010, 2014). 


\section{Results}

We use the publicly available MMT data (Table 1 of Brown et al. (2010)). The MMT stars could be either MS B stars, evolved BHB stars or blue stragglers (Brown et al., 2014). The Brown et al. (2010) data presents photometric and kinematic measurements of halo stars, with positions estimated both for the case the stars are BHB or blue stragglers, and the likelihood $0<f_{B H B}<1$ of each star being BHB. We take the BHB estimated distance if $f_{B H B}>0.5$, and the blue straggler distance if $f_{B H B} \leq 0.5$. Moreover, we remove the sub-sample of stars that have an estimated Galactic velocity $v>275 \mathrm{~km} \mathrm{~s}^{-1}$. Finally, we update the distances and velocities of this sub-population with the newer data in Table 1 of Brown et al. (2014).

It is important to note that stellar type plays a crucial role since the distances can be inferred only once the luminosities of the observed stars are known. To estimate luminosities and masses, the fitted stellar atmosphere parameters need to be compared to theoretical star tracks. Such a comparison can be done once the stellar type is assumed. As discussed, the cutoff in the distance-velocity distribution depends not only on the Galactic potential, but also the travel time, which depends on the stellar type. For MS stars, $t_{*}$ is directly related to the star mass $m_{*}$ by $t_{*} \propto m_{*}^{-\alpha}$, with $\alpha \approx 3$. However, only a few stars of the sample have a defined stellar type. For example, HVSs in the MMT survey are probably all MS B stars based on stellar rotation (Brown et al., 2014). Future identification of HVSs types will improve the constraint on the halo mass. Since $-\nu(r)$ has a two parameter dependence, we have to fix either the propagation time $t_{*}$ or $M_{D M}$ in order to draw critical lines in the v-r plane.

Figure 1 shows the critical lines for different halo masses and propagation times, along with the distribution in the v-r plane of the stars in the MMT sample. The bottom arc in Fig. 1 corresponds to the critical line $-\nu(r)$, whereas the top arc corresponds to $\nu(r)$. The critical lines show a clear dependence on $M_{D M}$ and $t_{*}$. The top figure shows the dependence on the halo mass, if $t_{*}=330 \mathrm{Myr}$ for all the stars. The zero-velocity point, i.e. the distance at which $-\nu(r)$ crosses the $\mathrm{x}$-axis, is an increasing function of the halo mass. In order to interpret these results, let us consider two different halo masses $M_{D M, 1}>M_{D M, 2}$ and a bound HVS. Once the baryonic content is fixed, $v_{e s c} \propto M_{D M}^{1 / 2}$. As consequence, $v_{e s, 1}>v_{e s, 2}$ and bound HVSs can be produced in the GC with a higher ejection velocity, since they have to satisfy the constraint $v_{e j}<$ $v_{\text {esc }}(r)$. Since $t_{*}$ is fixed by the stellar type, stars ejected in a Galaxy with $M_{D M, 1}$ are able to reach farther and fall back toward the GC in a shorter time, given their possible higher ejection velocities. Given that, the zerovelocity point is located at larger Galactocentric distances for heavier haloes. On the other hand, the shape of the critical lines depends also on the maximum HVS travel time. The bottom panel of Fig.1 shows the dependence on $t_{*}$, if the halo mass is fixed to $10^{12} \mathrm{M}_{\odot}$. The zero-
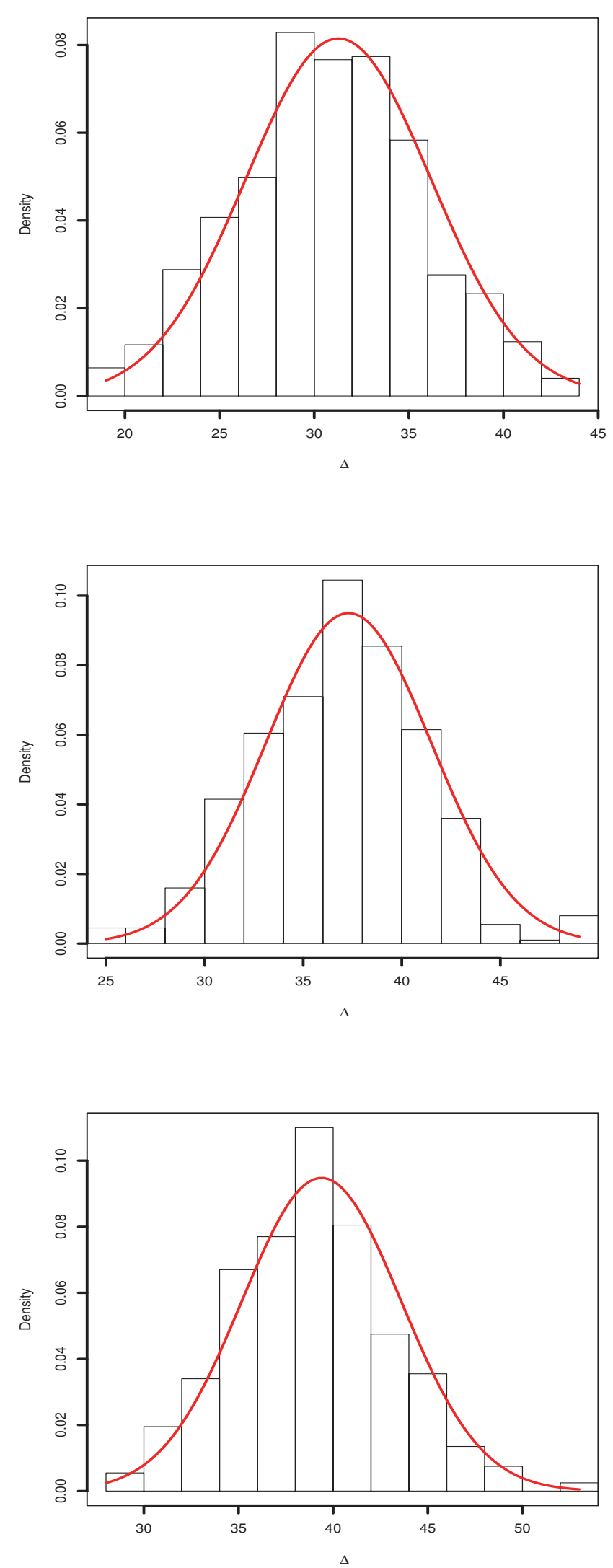

Figure 2: Distribution of the asymmetry $\Delta$ for the cases $M_{D M}=$ $0.6 \times 10^{12} \mathrm{M}_{\odot}($ top panel $), 0.7 \times 10^{12} \mathrm{M}_{\odot}($ central panel $), 0.8 \times 10^{12}$ $\mathrm{M}_{\odot}$ (bottom panel), when the travel time is fixed to $330 \mathrm{Myr}$. 
velocity point is an increasing function of the propagation time since stars are able to reach farther in their Galactic orbits within a longer $t_{*}$, before returning back to the GC with negative radial velocity. As discussed in the previous section, for MS stars the propagation time is directly related to the mass $m_{*} \propto t_{*}^{-\beta}$, with $\beta \approx 1 / 3$. Hence low-mass MS stars are able to reach larger distances in the Galactic halo within the MS lifetime, before returning back to the GC with negative radial velocity, compared to massive MS stars.

HVSs should present an asymmetric distribution. Although the clear cutoff in the distribution can be contaminated by the hot BHB stars of the sample, a statistical asymmetry between the number of ingoing and outgoing stars is still expected (Perets et al., 2009). We can search for the asymmetry $\Delta$ in the MMT stars sample by counting the number of stars beyond $\nu(r)$ and below $-\nu(r)$ for the different $M_{D M}$ and $t_{*}$. We define $\Delta_{+}$as the number of outgoing stars that lie beyond the critical line $\nu(r)$ plus the stars with positive velocities that have Galactocentric distances larger than the zero-velocity point. On the other hand, $\Delta_{-}$is defined as the number of ingoing stars that lie below the critical line $-\nu(r)$ plus the stars with negative velocities that have Galactocentric distances larger than the zero-velocity point. The asymmetry $\Delta$ is defined as $\Delta=\Delta_{+}-\Delta_{-}$.

As discussed, outside of the critical lines an asymmetry is expected because of the stars' finite lifetime and the MW potential. We vary $M_{D M}$ in the range of $[0.6-1.8] \times 10^{12}$ $\mathrm{M}_{\odot}$ and $t_{*}$ in the range of [300-640] Myr. For a fixed halo mass, we compute $\Delta$ for each value of $t_{*}$. In our calculations, we assume that all the stars have a maximal travel time $t_{*}$. This corresponds to the maximum travel time from the GC and determines, along with $M_{D M}$, the shape of the critical lines. In order to decide if a star lies below or beyond such lines, we compute error bars for distances and velocities from the data and generate random realizations inside the error bars. We take into account the fact that distances of MMT sample stars have large uncertainties, since they depend on the assumed absolute magnitude and stellar type. For the cases under analysis, we perform 1000 Monte Carlo realizations of $\Delta$ to propagate the uncertainties, and fit the resulting distribution with a normal function. Then, we associate the mean of the distribution with $\Delta$ and the standard deviation with the uncertainty in $\Delta$. Figure 2 illustrates the distribution of the asymmetry $\Delta$ for the cases $M_{D M}=0.6-0.7-0.8 \times 10^{12} \mathrm{M}_{\odot}$, when the travel time is fixed to $330 \mathrm{Myr}$ (see also Table 2). Figure 3 shows the resulting $\Delta$ for different halo masses as a function of $t_{*}$.

Brown et al. (2007a) found a significant excess of stars travelling with $v_{r}>275 \mathrm{~km} \mathrm{~s}^{-1}$. They compared the asymmetry in the velocity distribution of the halo stars to the theoretical predictions of Bromley et al. (2006), and concluded that HVSs ejected both on unbound and bound orbits are the most plausible explanation for the observed excess of positive-velocity outliers. Bound HVSs are stars
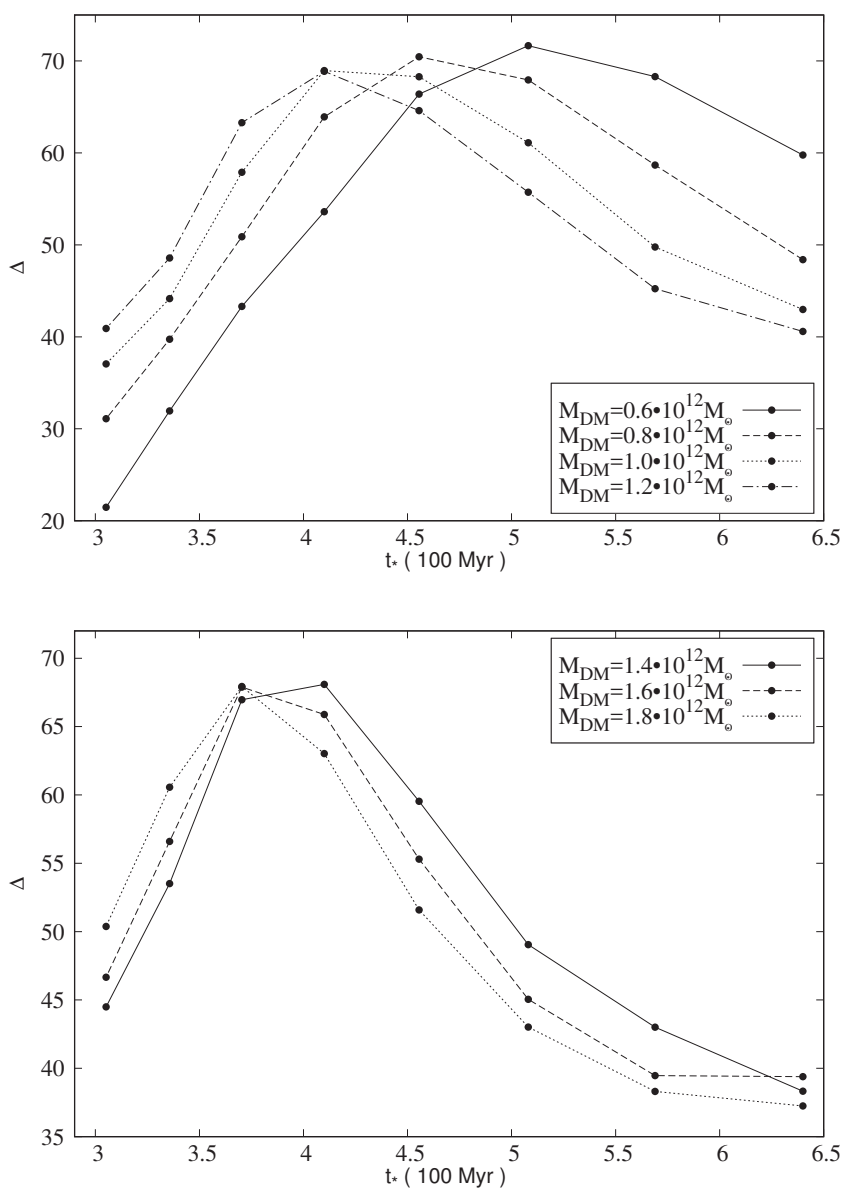

Figure 3: $\Delta$ for different halo masses as a function of $t_{*}$, for $0.6 \leq$ $\left(M_{D M} / 10^{12} \mathrm{M}_{\odot}\right) \leq 1.2($ top panel $)$ and $1.4 \leq\left(M_{D M} / 10^{12} \mathrm{M}_{\odot}\right) \leq$ 1.8 (bottom panel).

\begin{tabular}{|ccc|}
\hline$M_{D M}\left(10^{12} \mathrm{M}_{\odot}\right)$ & $\Delta$ & $\sigma_{\Delta}$ \\
\hline 0.6 & 31.28 & 4.89 \\
0.7 & 37.30 & 4.20 \\
0.8 & 39.38 & 4.21 \\
0.9 & 43.42 & 4.36 \\
1.0 & 44.60 & 4.07 \\
1.2 & 49.33 & 4.58 \\
1.4 & 53.31 & 4.65 \\
1.6 & 56.55 & 4.45 \\
1.8 & 60.57 & 3.98 \\
\hline
\end{tabular}

Table 2: Values of the asymmetry $\Delta$ in the case $t_{*}=330 \mathrm{Myr}$ 
ejected from the GC on bound orbits by the same mechanism that produces unbound stars, i.e. their ejection velocity does not exceed the local escape speed. Moreover, Brown et al. (2007a,b) showed that, while stars with velocities $\left|v_{r}\right|<275 \mathrm{~km} \mathrm{~s}^{-1}$ are well described by a Gaussian distribution, the asymmetry of stars with $v>275 \mathrm{~km} \mathrm{~s}^{-1}$ is significant at the $5 \sigma$ level. The choice of $275 \mathrm{~km} \mathrm{~s}^{-1}$ as threshold is motivated by the relative absence of stars with velocities less than $275 \mathrm{~km} \mathrm{~s}^{-1}$ in the MMT survey (Brown et al., 2007a,b, 2014). The lack of stars moving at $v_{r}<-275 \mathrm{~km} \mathrm{~s}^{-1}$ suggests that the bound positivevelocity outliers in the sample have lifetimes less than the orbital turn-around time. Brown et al. (2014) presented 37 stars that cause the excess of positive-velocity outliers (stars with $v>275 \mathrm{~km} \mathrm{~s}^{-1}$ in Table 1 ). Some of these outliers are unbound HVSs. By definition, a star is considered as an unbound HVS if its Galactocentric velocity exceeds the local escape velocity at its Galactocentric distance. Table 3 shows the number of HVSs, $\eta$, computed for different halo masses. Here $\eta$ depends on the assumed Galactic potential. Since $v_{e s c} \propto M_{D M}^{1 / 2}$, different halo masses give different number of stars beyond the local escape velocity, with more massive haloes predicting a lower number of HVSs. Given $\eta$ for $M_{D M}=10^{12} \mathbf{M}_{\odot}$, the Brown et al. (2014) data suggests that the number $N_{H V S}$ of unbound $2.5-4 \mathbf{M}_{\odot}$ HVSs is $\sim 100$ over the entire sky within $r<100 \mathrm{kpc}$. Taking into account $2.5-4 \mathbf{M}_{\odot}$ HVSs that do not survive to reach large distances, and considering a canonical IMF (Kroupa, 2001), Brown et al. (2014) estimated $\sim 300$ HVSs over the entire sky within $r<100 \mathrm{kpc}$ ejected with a rate of $1.5 \times 10^{-6} \mathrm{yr}^{-1}$. Based on the results presented in Table 3, the number of expected HVSs within 100 kpc would be $1.25 N_{H V S}$ if $M_{D M}=0.6 \times 10^{12} \mathbf{M}_{\odot}$ and $0.75 N_{H V S}$ in the case of $M_{D M}=1.8 \times 10^{12} \mathbf{M}_{\odot}$ for an NFW dark halo. This slight change in the predicted total number of HVSs is consistent with the uncertainties on the ejection rates of $\mathrm{HVSs}$ and on the binary population in the GC. Figure 4 shows the escape speed curves for different $M_{D M}$ along with the MMT positive-velocity outliers with $v>275 \mathrm{~km} \mathrm{~s}^{-1}$. As in the case of $\Delta$, we take into account the uncertainties in stellar distances. In order to decide if a star lies below or beyond $v_{e s c}$, we compute error bars for distances and velocities from the data and generate random realizations inside the error bars. We perform 1000 Monte Carlo realizations of $\eta$ to propagate the uncertainties, and fit the resulting distribution with a normal function. Then, we associate the mean of the distribution with $\eta$ and the standard deviation with the uncertainty in $\eta$.

As discussed above, Brown et al. (2014) presented $\Gamma=$ 37 stars that cause the excess of positive-velocity outliers at $v_{r}>275 \mathrm{~km} \mathrm{~s}^{-1}$, whose plausible explanation is outgoing bound and unbound HVSs (Brown et al., 2007a,b). The asymmetry in the velocity-distance distribution is due to outgoing unbound and bound HVSs outside the critical

\begin{tabular}{|cccc|}
\hline$M_{D M}\left(10^{12} \mathrm{M}_{\odot}\right)$ & $\eta$ & $\sigma_{\eta}$ & $\Gamma-\eta$ \\
\hline 0.6 & 24.40 & 1.04 & 12.60 \\
0.8 & 21.97 & 0.81 & 15.03 \\
1.0 & 20.20 & 0.85 & 16.80 \\
1.2 & 18.81 & 0.60 & 18.19 \\
1.4 & 17.90 & 0.75 & 19.10 \\
1.6 & 16.56 & 0.83 & 20.44 \\
1.8 & 15.28 & 0.84 & 21.72 \\
\hline
\end{tabular}

Table 3: Number of unbound $(\eta)$ and bound $(\Gamma-\eta)$ HVSs

lines region as a consequence of the cutoff in the ingoing HVSs. However, the clear cutoff in the ingoing stars may not be observable since it would be smeared by the halo stars contaminating the sample, but a statistical asymmetry between the number of ingoing and outgoing stars in the sample is still expected (Perets et al., 2009). Hence, the estimated value of the asymmetry $\Delta$ that quantifies the number of bound and unbound HVSs must be compatible with the number of outliers that give the excess in the velocity distribution $\Gamma$. Perets et al. (2009) suggested that another source for the asymmetry in the v-r plane may be the runaways from the Galactic disk (Silva \& Napiwotzki, 2011; Brown, 2015). However, the MMT survey targets the Galactic halo and covers high Galactic latitudes $|b| \gtrsim 30^{\circ}$, where the only source of contamination is hyper-runaways (Brown, 2015). Hyper-runaways are stars ejected from the Galactic disk, probably as a consequence of multi-body interactions or supernovae explosions (Irrgang et al., 2010), with Galactic rest-frame velocities of the order of the local escape speed. Heber et al. (2008) found that the Galactic rest-frame velocity of the massive $\mathrm{B}$ giant runaway $\mathrm{HD}$ 271791 was larger than the local escape speed. The ejection rate of hyper-runaways with speeds comparable with HVSs is $\approx 8 \times 10^{-7} \mathrm{yr}^{-1}$ (Brown, 2015). On the other hand, the ejection rate of HVSs is $\approx 10^{-5}-10^{-4} \mathrm{yr}^{-1}$ (Yu \& Tremaine, 2003). Since the ejection rate of hyperrunaways is $10-100$ times smaller than HVSs rate, we expect $\approx 1$ hyper-runaway each $\approx 10-100$ HVSs (Perets \& Sǔbr, 2012). Since this source of error is $\ll \Gamma$, we conclude that a possible contamination by hyper-runaways does not affect significantly our results. In conclusion, the favored model is the one for which $\Gamma$ is comparable to $\Delta$, within its error bars.

The asymmetry has a two dimensional dependence on $M_{D M}$ and $t_{*}$, which gives two possible interpretations depending on whether we fix the halo or the travel time. By fixing the halo mass we can estimate the preferred value of the travel time. As a consequence, since HVSs are probably MS stars and $m_{*} \propto t_{*}^{-\beta}$, we could evaluate the preferred mass of HVSs. On the other hand, by fixing the mass of the HVSs, we can constrain $M_{D M}$. Table 1 of Brown et al. (2014) presents also mass estimations for the unbound and bound populations of HVSs. The average mass of HVSs is $3.11 \mathrm{M}_{\odot}$. If the travel time of HVSs is fixed to $330 \mathrm{Myr}$ (see Table 2), which corresponds to the 


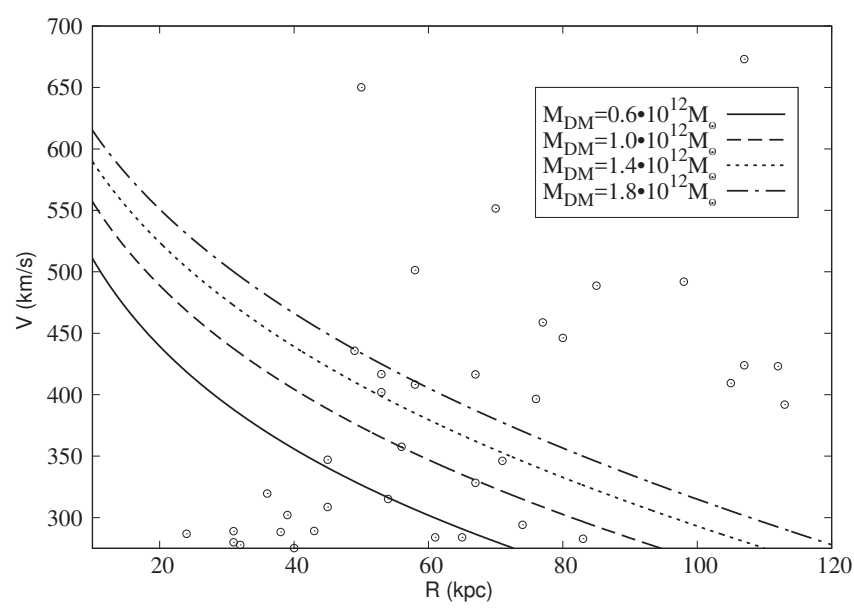

Figure 4: Escape velocity curves for different halo masses. Circles are observed distances and velocities of the MMT star sample for stars with $v>275 \mathrm{~km} \mathrm{~s}^{-1}$ (Brown et al., 2014).

average HVSs mass, the NFW halo mass parameter $M_{D M}$ is constrained to $(0.6-0.9) \times 10^{12} \mathrm{M}_{\odot}$, which gives a favored mass for the Milky Way in the range $(1.2-1.6) \times 10^{12} \mathrm{M}_{\odot}$ inside the virial radius $r_{200}$ (Klypin et al., 1999). Perets et al. (2009) suggest that an asymmetry of eight stars corresponds to the $1 \sigma$ probability level. In this case, $M_{D M}$ is constrained to $(0.6-1.2) \times 10^{12} \mathrm{M}_{\odot}$, which yields a Milky Way mass in the range $(1.2-1.9) \times 10^{12} \mathrm{M}_{\odot}$.

Gnedin et al. (2010) used the MMT halo stars data (without including the outliers in the sample, i.e. the HVSs) to derive the Galactic circular velocity and the MW mass distribution. Adopting a three-component Galactic potential (Klypin et al., 1999), they found that the Brown et al. (2010) data suggest a virial mass $M_{v i r}=$ $(1.6 \pm 0.3) \times 10^{12} \mathrm{M}_{\odot}$ at the virial radius $r_{v i r}=300 \mathrm{kpc}$. Our inferred MW mass range is consistent with the Gnedin et al. (2010) results and recent independent determinations (McMillan, 2011; Eadie et al., 2015; McMillan, 2016).

Our results can be improved with better data on the mass and 3D velocity of HVSs. The tracers we studied suffer from the lack of tangential velocity measurements since the MMT survey is a spectroscopic survey. Some of the HVSs may come from a nearby galaxy (Gualandris \& Portegies Zwart, 2007; Sherwin et al., 2008; Boubert \& Evans, 2016). If a large fraction of the HVSs originate from the Large Magellanic Cloud (LMC) (Gualandris \& Portegies Zwart, 2007), the method described in this paper would not be suitable. Gaia will be able to provide data for HVS proper motions, and hence tangential velocities, for some of the stars we considered and should be able to distinguish LMC HVSs from MW ones. The upcoming high-quality Gaia data will allow to improve the constraint on the MW mass distribution.

As discussed, the estimated masses of HVSs, and hence their lifetimes, play a crucial role in the determination of the their distances. To take into account the possible effects of the HVSs masses, we consider the error bars on the HVS masses (Brown et al., 2014) and estimate the smallest $\left(2.93 \mathrm{M}_{\odot}\right)$ and largest $\left(3.28 \mathrm{M}_{\odot}\right)$ average masses of HVSs. In such cases, the best fit $M_{D M}$ would be $\sim 1.4$ times larger and $\sim 0.7$ smaller, respectively, than the best fit $M_{D} M$ for the average HVSs mass $\left(3.11 \mathrm{M}_{\odot}\right)$. This would constrain $M_{D M}$ to $\approx(0.4-1.2) \times 10^{12} \mathrm{M}_{\odot}$. We note that $M_{D M} \gtrsim 0.5-0.6 \times 10^{12} \mathrm{M}_{\odot}$ from independent measurements (Wang et al., 2015), reducing our interval to $\approx(0.6-1.2) \times 10^{12} \mathrm{M}_{\odot}$. Finally, we note that if the Milky Way halo is constrained by an independent measurement, the results in this paper (Fig. 3) can be used to constrain the average lifetime and mass of the observed HVSs.

\section{Conclusions}

We have used the kinematics of HVSs as a method to constrain the MW mass. We studied the kinematics of HVSs observed by the MMT survey (Brown et al., $2010,2014)$ in the Galactic halo. The asymmetric velocitydistance distribution of the observed stars depends both on their lifetimes and the MW potential. We have found that, if the travel time of HVSs is fixed to $330 \mathrm{Myr}$, which corresponds to the average HVSs mass, the halo mass parameter is constrained to the range $(0.6-1.2) \times 10^{12} \mathrm{M}_{\odot}$, which gives a favored mass for the Milky Way in the range $(1.2-1.9) \times 10^{12} \mathrm{M}_{\odot}$ inside the virial radius $r_{200}$.

\section{Acknowledgements}

We thank Warren Brown for supplying the data on the kinematics of the stars in the HVSs sample and for helpful comments on the paper. We also thank Scott Kenyon and an anonymous referee for insightful comments. GF acknowledges hospitality from the Institute for Theory and Computation at the Harvard-Smithsonian Center for Astrophysics, where the early plan for this work was conceived, and also thanks David Merritt for useful discussions and comments. GF acknowledges Sapienza University of Rome for the grant "Progetti Avvio alla Ricerca" (Starting Research Grant, 0051276), which funded part of this work.

\section{References}

Boubert, D., Evans, N. W. 2016, 825, L6

Boylan-Kolchin, M., Bullock, J. S.,\& Kaplinghat, M. 2011, MNRAS, 415, L40

Boylan-Kolchin, M., Bullock, J. S., Sohn, S. T., Besla, G.,\& van der Marel, R. P. 2013, ApJ, 768, 140

Bovy, J., Bahmanyar, A., Fritz, T. K.,\& Kallivayalil N. 2016, ApJ, 833,31

Bromley, B. C., Kenyon, S. J., Geller, M. J., Barcikowski, E., Brown, W. R.,\& Kurtz M. J. 2006, ApJ, 653, 1194

Bromley, B. C., Kenyon, S. J., Brown, W. R.,\& Geller, M. J. 2009, ApJ, 706, 925.

Brown, W. R. 2015, ARA\& A, 53, 15 
Brown, W. R., Geller, M. J.,\& Kenyon, S. J. 2014, ApJ, 787, 89

Brown, W. R., Geller, M. J.,\& Kenyon, S. J.,\& Diaferio, A. 2010, AJ, 139,59

Brown, W. R., Geller, M. J., Kenyon, S. J.,\& Kurtz, M. J. 2005, ApJ Lett., 622, L33

Brown, W. R., Geller, M. J., Kenyon, S. J., Kurtz, M. J.,\& Bromley, B. C. 2007, ApJ, 660,311

Brown, W. R., Geller, M. J., Kenyon, S. J., Kurtz, M. J.,\& Bromley, B. C. 2007 , ApJ, 671, 1708

Capuzzo-Dolcetta, R.,\& Fragione, G. 2015, MNRAS, 454, 2677

Deason, A. J., Belokurov, V., Koposov, S. E.,\& Rockosi, C. M. 2014 ApJ, 787, 30

Eadie, G. M., Harris, W. E.,\& Widrow, L. M. 2015, ApJ, 806, 54

Fragione, G.,\& Capuzzo-Dolcetta, R. 2016, MNRAS, 458, 2596

Fragione, G., Capuzzo-Dolcetta, R.,\& Kroupa, P. 2016, preprint arXiv: 1609.05305

Fragione, G..\& Ginsburg, I. 2017, MNRAS, 466, 1805

Gibbons, S. L. J., Belokurov, V.,\& Evans, N. W. 2014, MNRAS, 445, 3788

Ginsburg, I.,\& Loeb, A. 2006, MNRAS, 368, 221

Ginsburg, I.,\& Loeb, A. 2007, MNRAS, 376, 492

Ginsburg, I., Loeb, A.,\& Wegner, G. A. 2012, MNRAS ,423.1, 948

Gnedin, O. Y., Gould, A., Miralda-Escudé, J.,\& Zentner, A. R. 2005, ApJ, 634, 344

Gnedin, O. Y., Brown, W. R., Geller, M. J.,\& Kenyon, S. J. 2010, ApJ Lett., 720, L108

Gould, A.,\& Quillen, A. C. 2003, ApJ, 592, 935

Gualandris, A.,\& Portegies Zwart, S. 2007, MNRAS Lett., 376, L29

Guo, Q., Cooper, A. P., Frenk, C., Helly, J.,\& Hellwing, W. A. 2015, MNRAS, 454, 550

Heber, U., Edelmann, H., Napiwotzki, R., Altmann, M.,\& Scholz, R. D. 2008, A\& A, 483, L21

Hills, J. G. 1988, Nature, 331, 687

Irrgang, A., Przybilla, N., Heber, U., Nieva, M. F.,\& Schuh S. 2010, ApJ, 711, 138

Kallivayalil, N., van der Marel, R. P., Besla, G., Anderson, J.,\& Alcock, C. 2013, ApJ, 764, 161

Kang X., Wang L.,\& Luo Y. 2016, MNRAS, 460, 2152

Kenyon, S. J., Bromley, B. C., Geller, M. J.,\& Brown, W. R. 2008, ApJ, 680, 312

Kenyon, S. J., Bromley, B. C., Brown, W. R.,\& Geller M. J. 2014, ApJ, 793, 122

Klypin, A., Kravtsov, A. V., Valenzuela C.,\& Prada F. 1999, MNRAS, 522, 82

Kroupa, P. 2001, MNRAS, 322, 231

Küpper, A. H., Balbinot, E., Bonaca, A., Johnston, K. V., Hogg, D. W., Kroupa, P.,\& Santiago, B. X. 2015, ApJ, 803, 80

Li, Y., et al. 2015, 15.8, 1364

Navarro, J. F., Frenk, C. S.,\& White, S. D. 1997, ApJ, 490, 493

McMillan, P. J. 2011, MNRAS, 414, 2446

McMillan, P. J. 2016, preprint (arXiv:1608.00971)

O'Leary, R. M.,\& Loeb, A. 2008, MNRAS, 383, 86

Palladino, L. E., et al. 2014, ApJ, 780, 7

Perets, H. B., et al. 2009, ApJ, 697.2, 2096

Perets, H. B.,\& Sǔbr, L. 2012, ApJ, 751, 133

Reid, M. J., et al. 2014, ApJ, 783, 130

Sari, R., Kobayashi, S.,\& Rossi, E. M. 2010, ApJ, 708, 605

Sherwin, B., Loeb, A.,\& O'Leary, R. M. 2008, MNRAS, 386, 1179

Silva, M. D. V.,\& Napiwotzki, R. 2011, MNRAS, 411, 2596

Taylor, C., Boylan-Kolchin, M., Torrey, P., Vogelsberger, M.,\& Hernquist, L. 2016, MNRAS, 461, 3483

Wang, W., Han, J., Cooper, A. P., Cole, S., Frenk, C.,\& Lowing, B. 2015, MNRAS, 453, 377

Yu, Q.,\& Madau, P. 2007, MNRAS, 379, 1293

Yu, Q.,\& Tremaine, S. 2003, ApJ, 599, 1129

Ziegerer, E., Volkert, M., Heber, U., Irrgang, A., Gänsicke, B.T.,\& Geier, S. 2015, A\& A, 576, L14 